\title{
Understanding the Business Value of Social Information Systems - Towards a Research Agenda
}

\author{
Rainer Schmidt \\ Munich University of Applied \\ Sciences \\ $\underline{\text { rainer.schmidt@,hm.edu }}$
}

\author{
Kathrin Kirchner \\ Technical University of \\ Denmark \\ kakir@dtu.dk
}

\author{
Liana Razmerita \\ Copenhagen Business School \\ 1ra.msc@.cbs.dk
}

\begin{abstract}
Recent studies confirmed the creation of value by social information systems. However, few is known about the mechanisms that create value in social information systems. Using a design science approach, this article investigates how social information systems create value. Based on a literature review, we identify four types of emergent interactions in social information systems that create value: social production, co-creation, weak ties, and egalitarian decisions. The paper proposes a holistic framework for understanding business value and develops a research agenda to further explore the value creation mechanisms of social information systems.
\end{abstract}

\section{Introduction}

Social information systems comprise of a large variety of software used by organizations including social networking platforms, collaborative project management tools, or online/content communities [59]. They differ from traditional information systems by enabling emergent interactions [3]. Emergent interactions are interactions that are defined during run-time by two or more stakeholders. No plan or approval from a supervisor or management is necessary. Emergent interactions enable the articulation of personal into collective knowledge thus representing mechanisms for harnessing collective intelligence in the digital age $[7,51]$.

By enabling emergent interactions, social information systems differ fundamentally from, the prevailing information systems, so-called Tayloristic information systems. They are named in this way because they follow the ideas of Taylorism [42, 65]. Within Tayloristic information systems, users are only capable of interacting according to specific features and design fixed in software. They may initiate these interactions on their own. However, they are bound to very limited types defined in the information systems (e.g., most

URI: https://hdl.handle.net/10125/64063

ERP systems that allow their users a predefined set of transactions only).

Business value of a social information system is traditionally defined as the pure economic value generated by the system (e.g., ROI). However, it may comprise additionally non-monetary value for employees, customers, business partners, or even society. Business value is important for measuring success, understanding the benefits, and designing an associated strategy. It has been shown that information technology and systems, in general, contribute to the improvement of organizational performance $[10,17]$. An integrative model of IT business value is presented in [45]. KPIs and balanced score cards are also approaches to improve the measurement of value created by information technology[34]. However, these approaches refer to Tayloristic information systems but not to social information systems.

The literature on business value of social media technologies is fragmented to specific application domains as for example: innovation communities [18], a specialized investigation into the ROI in hotel industry [11], the ROI of social media [24, 64], or brand and firm risk is done in [57]. A similar analysis on firm performance is presented in [64]. The impact of social paradigms on business processes has been investigated in [60] and [9]. The Social Media business value compass was introduced to quantify the effects of social platforms (e.g. Yammer, Podio, Slack) and assess the business value derived from the digitalization [38].

This and further research provide evidence that social information systems create value in very different forms. However, previous research also has pointed to the fact that the why and how in the research on the business value of IT are insufficiently researched [1]. In particular, the need to intensify the research on social computing was identified in [6]. Therefore, we will investigate how social information systems differ from Tayloristic information systems enabling the creation of value. This investigation is part of longer, ongoing research. Our research question is: "How value is created 
in social information systems and what value creation mechanisms should be further investigated?"

The contribution of this paper is to identify the value-creating mechanisms in social information systems and to create an agenda that identifies areas that need further research in order to understand the mechanism of value creation in social information systems.

Research on the business value of IT requires at least two components: 1 . an IT (management) variable or its manifestation. 2. an endogenous variable influenced by IT [39]. This also applies to social information systems.

We use a design science approach to develop a business value framework and a research agenda as an artifact [28]. Understanding the business value is relevant because it allows explaining real-world phenomena associated with the use of social information systems. The evaluation is done using an informed argument.

Our paper is structured according to the recommendations for design science research projects [28]. First, we demonstrate the relevance of our research by connecting the context of the research project with the design science activities [54]. We then enter the rigor cycle by performing a systematic literature review [54]. We enter the design cycle by deriving a business value framework as a model type artifact [54]. The business value framework abstracts different types of value created by social information systems, presented in section three. The framework also abstracts four types of emergent interactions (as endogenous variables) that create one or several types of value (elaborated in section four). In section five, we describe how these interactions create one or more types of value differentiated in section three. Based on this analysis, we identify areas of research and develop the research agenda in section six. Finally, we give a conclusion and outlook on our ongoing research.

\section{Background - From Tayloristic to Social Information Systems}

For a long time, most organizations and IT systems were designed based on Tayloristic principles [42, 65]. In a top-down manner, the management of an organization splits complex tasks into simple ones, assigns them to employees, measures the success and pays the employees accordingly [72]. Tayloristic principles are still in use or even thriving in some companies and are applied even to white-collar workers [16].

In this setting, information systems serve to assign the tasks resulting from these processes to human task carriers and to supervise their execution. They create value by automating the coordination between tasks and thus improving the efficiency, quality and speed of execution while replacing human bureaucrats.
The first approaches for social information systems had a strong counter-culture attitude based on the tayloristic structures in society [23]. Social information systems break with the hitherto Tayloristic approach of information system design [59], due to the advent of the internet and its associated Web 2.0 principles [48].

A Tayloristic information system does not allow the individual user to define new types of interactions. The interactions in tayloristic information systems are predefined. However, they support the creation of new instances of predefined interaction types as part of business processes, e.g. an individual service request.

The differences between social information systems and Tayloristic information systems can also be described by using the five views of the Architecture of Integrated Information Systems [56]. The functions of social information systems can be extended a run-time by the users - contrary to Tayloristic information systems. Social information systems allow users to personalize the interaction, to modify the workflow and business processes, by associating additional users and granting privileges to them in a decentralized manner. E.g. in social networks, wikis, blogs the user may decide to whom the content created by him is visible. Users of social information systems can create new data structures or modify the existing data model, e.g., in wikis. Social information systems also provide the capability to support user-defined processes. By combining these capabilities, the new services and products can be created by the user.

Emergent interactions are often bidirectional. Bidirectional interactions reflect the more egalitarian thinking in social information systems that contrasts with the dominant unidirectional interaction in Tayloristic information systems, reflecting the chain of command.

Social information systems appear in a multitude of architectures nowadays. The most important types are social networking sites, wikis, blogs, and content communities.

Social networks are defined as the computer-based connection of people and organizations [25]. Social networking sites (SNS) allow the individual to construct a public or semi-public profile within a bounded system [8]. Furthermore, they enable the user to articulate a list of other users with whom they share a connection and to view their activities [8]. Enterprise SNS (e.g., Yammer, Workplace) are dedicated to support communication and networking within organizations. Professional SNS allow individuals to build an identity, a reputation and maintain professional relationships through online interactions. Different types of social media are differentiated according to the social presence and self-presentation or self-disclosure in [33].

Wikis support collaborative editing of Web page content enabling simple, distributed, and traceable 
changes [27]. Wikis offer easy to set-up tools for the collaborative editing of multimedia texts and means for integrating the individual contributions into the already existing results [22]. In an organizational context, wikibased systems have been adopted to aggregate projectrelated knowledge, best practices, or lessons learned [27].

An important difference of wikis to many other kinds of social information systems such as blogs is the merging of content. That means content entered by users is merged with the content of other users in a way that it becomes integrated [22].

Blogs are online journals consisting of discrete entries typically displayed in reverse chronological order, so the most recent post appears first; they are used to chronicle the lives and opinions of their authors [40]. Microblogs like Twitter enable users to send/read short message in a message stream (social network) that others can follow. It is used by people to communicate, advertise events, seek or share information [40].

Content communities or online communities contain information in the form of text, voice, image, or video (e.g., via YouTube, Instagram, Podcasts); everybody can share information, rate, or comment on the content provided by the community.

Social information systems can be regarded as social platforms [2]. They provide three basic functions: the attraction of producers and consumers, the matching of producers and consumers and the facilitation of the interactions between producers and consumers [49]. Platforms can be considered as multi-sided markets as they have a special capability to orchestrate external resources [21]. They provide both direct and indirect network effects. Direct network effects appear on the same side of the market; indirect network effects are created across different sides of the market.

The business value of social information systems can be only fully reaped in an organization if the use of the systems is aligned with the business strategy, organizational goals, and culture. According to [35] five categories have to be considered that form a star model (Figure 1):

- Strategy: The strategy gives the company a direction by stating goals and explain the business value and the sources of competitive advantage.

- Structure: The structure category explains how decision power is distributed in the company, which can be done, e.g., via specialization, distribution of power, and departmentalization.

- Processes: Information and decision processes are embedded in the organizational structure.

- Rewards: Incentives can be given if employees behave in a way that they support the strategic aims of the company.
- People: This category determines skills and mindsets of employees that influence recruitment, training, or development of employees.

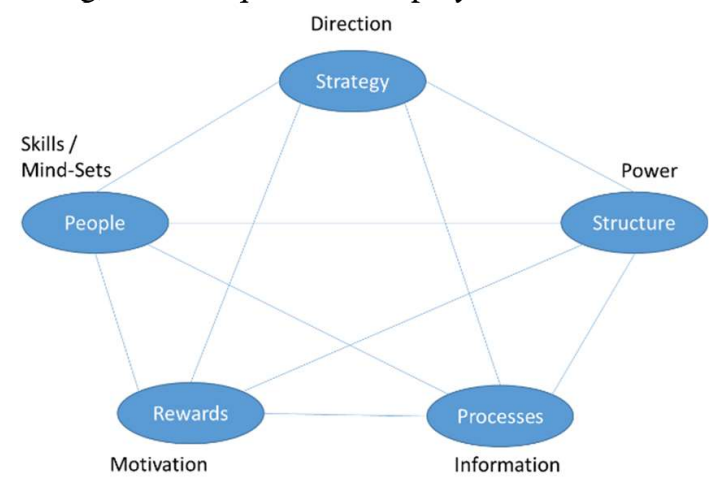

Figure 1: Star Model [35]

\section{Business Value of Social Information Systems}

Following the recommendations for design science research projects, we demonstrated the relevance of our research in the previous sections [28]. We will now enter the rigor cycle by investigating the existing knowledge base activities [54]. Our findings are based on an extensive, structured literature review of research because the latest comprehensive literature review dates from 2011 [58]. As a guidance for our review we used the recommendations in [69] and [47]. Regarding the selection of the papers, we considered the list of publications considered relevant in [70]. We queried leading scientific databases including ACM Digital Library, AISel, IEEExplore, SpringerLink, ScienceDirect, Google Scholar. To broaden the number of papers found we used the search terms social information system, social software, social computing social informatics, social media, social collaboration, social networks, social production, social web and Web 2.0 in connection with business value. In this way we found 312 papers. 15 of them could be excluded because of irrelevance. We used forward and backward search as proposed in [47] to augment the number of sources. In this way, we could include papers that are highly influential due to their high citation count, but not within the formal scope.

A comprehensive model of IS business model value is presented in [61]. Its components are IT investments, Non-IT investments, context factors, lag effects, and performance. Based on this model we investigate social information systems as assets. Our scope is their impact on process performance but we also consider context factors. Understanding how information technology can create business value is complex and challenging [12]. There are different measures for the business value of information technology. The measures productivity, 
business profitability, and consumer surplus for the business value of IT are introduced in [29]. In order to create business value, IT should be aligned to organizational strategy [19], company processes [37], organizational structure [36], organizational culture [31] and operational aspects [68].

The value of social information systems is difficult to quantify in economic terms like a return on investment. Business value does not come from the platform as such but from how the platform is used. With social information systems, virtual customer environments are created that generate business value besides of e-commerce when customers interact with the company, cocreate content, and share power [14]. A metric called "return on contribution" is suggested to measure the usage of social information systems [46]. It is defined as the number of people benefitting from a resource divided by the number of people that create or contribute to that resource. A success measurement framework taking into account different actions (search, edit, rate, label, clarify, notify, share) and the two success measurement dimensions usage and business value is proposed in [55]. Business value can be measured, e.g., by reduced time to find information, increased quality of content, reduced amount of emails, generated a number of ideas, or reduced travel costs.

The business value of a social system is different for different user types, e.g., depending if a person only consumes knowledge and information or also contributes to new knowledge creation. Furthermore, the business value of a specific system is influenced by the quality of provided information, perceived usefulness and usability. Such benefits can be measured on the individual or the organizational level.

The perceived business value of enterprise social information systems is classified into four categories [38]: efficiency, innovation, retention of members, and knowledge and transparency. The compass allows organizations to orient their strategy in order to increase the associated value. These categories are explained in the following subsections.

\subsection{Efficiency}

Efficiency describes how well or how productively work processes are enabled by social information systems in a company. A higher level of efficiency is one of the most important business value factors associated with the usage of social information systems [38]. The number of internal emails can be reduced [41], interaction and knowledge sharing improved [53], communication becomes faster and more efficient, and new employees can be easier integrated because they get access to all knowledge resources [38].

\subsection{Innovation}

Social information systems can lead to increased knowledge sharing, idea generation [71], and innovation. The involvement of a company-internal or external crowd may lead to new ways of acquiring knowledge, ideas, co-creation, higher creativity, and joint problem solving [38]. Social information systems can lead to increased knowledge sharing, innovation activities, and idea generation $[38,71]$. The implementation of open innovation principles within organizations should allow knowledge to flow in and out of the organization. The adoption of open innovation principles requires a multistep organizational change process [13].

\subsection{Retention of Members and Knowledge}

Retention can be considered along two dimensions: retention of employees or, more generally, members of the social information systems and the retention of knowledge that is externalized through interactions and "online communal conversations" [43] which would otherwise be lost. Members can connect easier via a social platform and get to know each other even if they are not sitting in nearby offices [38]. Freelancers, distance workers that are traveling or working from home can be better integrated, so they do not feel isolated and can share experiences. Thus, collaboration between employees as well as between departments and teams is improved [41]. Online platform connectivity and interactions may lead to a "strengthening of the weak" ties between employees and thus lead to an improved social capital and retention of employees. They get quicker access to knowledge [71], information can be found easier, and conversations are saved [38].

\subsection{Transparency}

The use of social information systems facilitates the exchange of information across silos and distribute them between different teams, departments, and geographical locations. Employees can improve the visibility of their knowledge, skills, and ideas independent of their position in the company's hierarchy. This way, employees can engage more and be included in decision processes [38]. Therefore, transparency changes company culture.

\section{Value Creating Emergent Interactions}

Based on the literature review and as part of ongoing research, we found four value-creating emergent interactions. Social production, co-creation, weak ties, and egalitarian decisions. Future work may find additional ones or proof of completeness. 


\subsection{Social Production}

Social production can generally be defined as a productive economic activity carried out primarily for social and psychological purposes rather than financial remuneration [62]. People do not have monetary gains but are driven by satisfaction, building social capital, and reputation (social currency) [5]. Such contributions can be used for demonstrating expertise that can be helpful to find new jobs, as shown in [4]. Social production can replace market-based mechanisms for production coordination [5]. Benkler shows that social production enables the effective and efficient allocation of resources such as 'human creativity, time, and attention' [5].

Social production empowers people independent of their qualifications and position in the organization. It brings an alternative way to organize and collaborate [9]. Instead, production is organized by contributions designed and planned in a bottom-up manner [5].

The differences to other approaches become obvious in quality control, too. Instead of using the a-priori approach of Taylorism, checking the fulfillment of pre-defined criteria is important. Social production uses an aposteriori approach [5]. By making the contributions public, negative quality impacts the social reputation of the contributor. This may lead to more thorough quality control, because of not only the factors imposed by the hierarchy as with Taylorism, but also new, but relevant ones are taken into account [22]. Social production is supported by collaborative tools such as Wikis [27]. Wikis offer tools for the collaborative editing of multimedia texts and means for integrating the outcome into the already existing results.

\subsection{Co-Creation}

Co-creation can be defined as an emergent interaction or knowledge collaboration between both internal and external members (customers or stakeholders) of an organization [67]. Co-creation breaks the centralized, hierarchical approach of an organization and fosters a decentralized approach. Co-creation implies the involvement of end-users or stakeholders into the creation of products and services and innovation. On the contrary, social production focusses on the coordination of resources in the production of knowledge, software, programming capabilities. An example of co-creation is the implementation of the suggestion boards of software vendors.

\subsection{Egalitarian Decisions}

Egalitarian decisions break with the assumption of Tayloristic organizations that the decision competency is concentrated at the top of hierarchies or within experts
[63]. Instead, the bias in decisions shall be minimized by combining a multitude of single decisions. Egalitarian decision mechanisms are implemented in recommender systems such as Yelp. Everybody has the same right to contribute.

\subsection{Weak Ties}

The fourth characteristics is based on social network theory differentiation between strong and weak ties [26]. Decentralized open communities may foster collaboration between members who do not necessarily know each other but are connected through weak ties or who do not have any direct association. Weak ties break this paradigm by allowing and fostering associations between individuals and enable more fluid, flexible structure of an organization [26]. Weak ties are implemented in the matching mechanisms of social networks and platforms, e.g., based on properties of the users fitting well together, receive the suggestion to get into contact [26].

\section{Business value generation}

According to the recommendations for design science research projects [28], we have derived the business value framework as a model type artifact [54]. It is depicted in figure 2 .

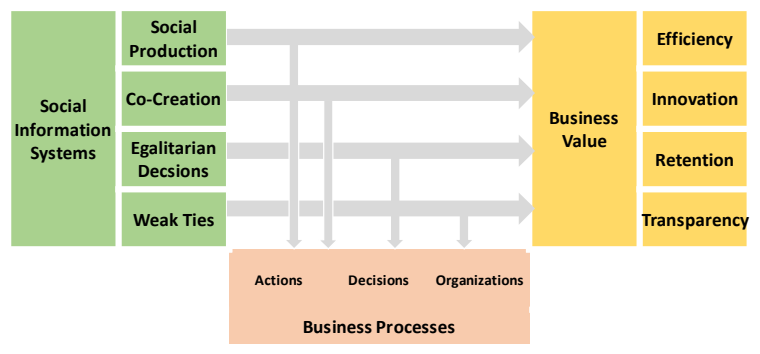

Figure 2: Business Value Framework

The foundation for our framework is the abstract synthesized IS business value model in [61]. Following this model, we consider social information systems as IS asset, that impacts process performance which in turn drives the organizational performance. We apply the definition of business processes in [20] and understand business processes as the creation and changing of artifacts in several half-ordered actions that are controlled by decisions. These actions may be in temporal or causal relations. Connected with actions are human actors that are part of an organization. Therefore, we will investigate how emergent interactions impact actions, decisions, and organizations. This discussion is used to de- 
scribe value-creating emergent interactions in more detail and to create a connection with the empirically identified business value generation. The following figure visualizes this relationship.

\subsection{Social Production}

Social production and co-creation provide new means for actions in business processes [11]. They enable the creation and editing of artifacts like hypertext, long and short documents or software code in new ways and thus providing value [15]. Wikis are the most important example of social information systems supporting hypertext [27]. They allow the creation and editing of documents of any length that may be linked without an ordering dimension. Blogs and microblogs also enable the creation and editing of documents and facilitate the flow of communication within organizations [40] [14]. Micro-documents are documents of a fixed maximum length [40]. Contrary to wikis, no fusion of the user inputs takes place.

Based on these mechanisms, social production accelerates communication by allowing an open group of contributors to work with an artifact [27]. Instead of running through an administrative procedure to get access, the contributors get immediate access. This also reduces the number of e-mails. By reducing the threshold to contribute new ideas, knowledge sharing is improved [33]. Also, access to experts is facilitated.

Social production improves innovation by enabling co-creation through crowdwork or crowdsourcing [30]. The open set of contributors improves the heterogeneity of backgrounds and thus increases creativity and improves problem-solving [14]. Furthermore, the retention of members and knowledge is improved. The sharing of experiences and learning are facilitated. Additionally, team building is improved by reducing the threshold for cooperation.

Social production also increases transparency. Contributors become visible independent of position. This and the inclusion into the decision process improves engagement. The transparency in social production also improves the accessibility of knowledge and communication across silos.

\subsection{Egalitarian Decisions}

Egalitarian decisions in social information systems provide several new types of decision support in business processes [44]. Rating systems provide both categorical information on items such as shared digital assets or products. Review systems offer non-categorical information [11]. By providing a summary of ratings and reviews, rating and review systems provide aggregated information on one item, but they do not put them into relation to other items. Ranking systems use ratings and reviews to compare items and create an order on them [51]. However, they use only one dimension. Recommendation systems go further and use multiple dimensions to create their recommendations.

Business value is created by egalitarian decisions by improving innovation, involvement of employees and by increasing creativity [57]. Furthermore, the retention of team members and their knowledge is improved [51]. Egalitarian decisions also create business value by improving transparency by increased visibility independent of positions and inclusion into the decision process. At the same time, communication across silos is improved.

\subsection{Weak Ties}

Weak ties impact business processes by their ability to create new relationships between employees and organizational structures [50]. More advanced social information systems may connect individuals by transitive relations to a person such as "friend of friends" or through recommendations. The most far-reaching concept is association by attributes. With these means also a person completely unconnected may get into contact.

Weak ties can potentially create business value by improving the flow of knowledge, the connectivity of employees, by increasing knowledge sharing, and the access to experts [66]. Weak ties improve connectivity and networking between employees and facilitate finding information. Transparency is increased by improved engagement and inclusion in the decision process. Particularly communication across silos is improved.

\section{Research Agenda}

Companies need to be ready to address challenges, transformations, or "unintended consequences" in relation to the introduction and use of social information systems [32]. From our point of view, black-box thinking is not sufficient to develop a methodology of how social information systems can successfully be used in a company to be valuable. We argue that emergent interactions social production, co-creation, egalitarian decisions, and the strengthening of weak ties can be considered as new ways to develop and capture value in social information systems. However, our research and the literature review also revealed several open research questions. An organization can only make fully use of the potential of social information systems if we address the strategic alignment. We suggest a research agenda taking into account both strategic alignment and business value considerations. 


\subsection{Strategic Alignment}

Using the categories proposed in the Star model [35], we can derive the following recommendations:

- People: Social information systems can be only useful if a sufficient number of employees use the system on regular basis by both contributing to the content, but also using the pieces of knowledge, interacting or connecting to others. This might also include training people how and when to use the systems and to build trust among employees.

- Rewards: Employees should be recognized or rewarded if they contribute and create value using social information systems. Organizational culture and leadership team should nurture an open and knowledge sharing culture. An earlier study found factors that influence social media communication within organizations identified that both intrinsic, as well as extrinsic motivational factors (recognition, monetary rewards), could influence the knowledge sharing the behavior of employees [52].

- Processes: Information and decision processes need to be changed. Decisions could be community decisions. Information should become available not only to a closed group but instead; employees should be able to share information regardless of position and department.

- Structure: An organization cannot follow a strict hierarchical decision-making structure, but instead become more transparent, overcome silos, and be open to proposals from all employees.

- Strategy: The use of social information systems has to be aligned with the strategy of the company and generate business value.

\subsection{Business Value - A Research Agenda}

Business value has both quantifiable and less quantifiable measures (e.g. satisfaction or retention of the employees, organizational learning) that must be considered. Business value can be assessed using "scientific management" approaches similar to Tayloristic approaches, but it could also be derived taking into consideration "unquantifiable human factors such as values meaning and experiences" [46:41]. Therefore, the following research questions remain still open and are part of a future research agenda:

- The currently identified value-creating emergent interactions of social information systems do not influence all identified aspects of business value. What other additional drivers of business value creation can be considered?
- Is any type of associated methodology that helps to improve productivity in the social systems and align them with the strategic aims of the company?

- Which kind of interventions could be used to foster interactions if a certain dimension of the business value should be strengthened?

- How can organizations leverage social information systems and redesign current work processes to focus on increasing the business value (e.g. in productivity or cost efficiencies) and define new work processes that extend beyond business value to include value, meaning, and engagement for their users (or stakeholders)?

- How can users of social systems be motivated and retained to participate?

- How does the use of a specific social information system will influence the organization as described in the star model?

- How can we measure the value created by emergent interactions in a quantitative way? Are there also interactions providing no or negative value? How can they be detected and mitigated?

\section{Conclusion}

Recent research showed that social information systems provide value to corporations and also differentiated forms of value creation [38]. However, only a few studies so far have investigated the mechanisms that lead to accomplishing value creation. Therefore, we started our research from the definition of social information systems as information systems that enable emergent interactions. That means these interactions are initiated by individuals on their own and not imposed by management, hierarchy, officer etc.

Our paper has identified four emergent interactions in social information systems that are drivers of business value. Social production organizes the creation of products and services by enabling contributions of individuals without requiring that these contributions are part of a top-down developed plan from management or administration. Co-production integrates the customer into the creation and change of products the services. Weak ties are relationships between individuals created on their own. These relationships cross the formal boundaries of the organization. Egalitarian decisions can be based on the votes, ratings or opinion of individuals in a democratic way. We analyzed how these value-creating emergent interactions are integrated into different types of social information systems.

Based on our findings, we developed a research agenda to explore value creation in social information systems. The research agenda contains objects both from strategy and business value research. Important 
objects of future research in information systems are methodologies that help to improve productivity in the social information systems and align them with the strategic aims of the company. The research agenda and the work of other researchers and our team on the research agenda will help to increase the value provided by social information systems.

Our research helps practitioners to make better decisions regarding the architecture of information systems. By reflecting on the question of whether a Tayloristic or Social Information System is needed to fulfill certain needs if the requirements are clarified. Understanding the business value of social information systems can help to overcome skepticism toward the systems and help to align their strategic use with the company's goals and use them more efficiently. Furthermore, our research framework improves the requirements elicitation for designing social information systems by identifying possible options in the form of emergent interactions.

As no research is without certain limitations, our findings have some limitations as well that are also tasks for further research. First, we encourage further investigation of value-creating emergent interactions, because we did not find arguments, why the four interactions found, are already complete. Second, empirical verification of our findings is an additional task for future work. Third, the four emergent interactions are applicable and function well in online communities such as (e.g. GitHub, Trip Advisor) however, there are potential tensions that might emerge in specific organizational context (e.g. large bureaucracies might not be agile enough to embrace changes or might not be ready to implement them).

\section{References}

[1] Agarwal, R., and H.C. Lucas, "The Information Systems Identity Crisis: Focusing on High-Visibility and High-Impact Research", MIS Quarterly 29, 2005, pp. 381-398.

[2] Alstyne, M.W.V., G.G. Parker, and S.P. Choudary, "Pipelines, Platforms, and the New Rules of Strategy", Harvard Business Review 94(4), 2016, pp. 54-62.

[3] Alter, S., Information systems, Addison-Wesley Longman Publishing Co., Inc., 1998.

[4] Benkler, Y., "Coase's Penguin, or, Linux and" The Nature of the Firm"', Yale Law Journal, 2002, pp. 369-446.

[5] Benkler, Y., The Wealth of Networks : How Social Production Transforms Markets and Freedom, Yale University Press, 2006.

[6] Bernstein, M.S., M.S. Ackerman, E.H. Chi, and R.C. Miller, "The trouble with social computing systems research",
Proceedings of the 2011 annual conference extended abstracts on Human factors in computing systems, 2011, pp. 389-398.

[7] Bhatt, G.D., "Management strategies for individual knowledge and organizational knowledge", Journal of knowledge management 6(1), 2002, pp. 31-39.

[8] Boyd, D.M., and N.B. Ellison, "Social network sites: Definition, history, and scholarship", Journal of computer-mediated Communication 13(1), 2007, pp. 210-230.

[9] Bruno, G., F. Dengler, B. Jennings, et al., "Key challenges for enabling agile BPM with social software", Journal of Software Maintenance and Evolution: Research and Practice 23(4), 2011, pp. 297-326.

[10] Brynjolfsson, E., and L. Hitt, "Paradox lost? Firm-level evidence on the returns to information systems spending", Management science 42(4), 1996, pp. 541-558.

[11] Buhalis, D., and E. Mamalakis, "Social media return on investment and performance evaluation in the hotel industry context", In Information and Communication Technologies in Tourism 2015. Springer, 2015, 241-253.

[12] Cao, G., Y. Duan, T. Cadden, and S. Minocha, "Systemic capabilities: the source of IT business value", Information Technology \& People 29(3), 2016, pp. 556-579.

[13] Chiaroni, D., V. Chiesa, and F. Frattini, “The Open Innovation Journey: How firms dynamically implement the emerging innovation management paradigm", Technovation 31(1), 2011, pp. 34-43.

[14] Culnan, M.J., P.J. McHugh, and J.I. Zubillaga, "How large US companies can use Twitter and other social media to gain business value.", MIS Quarterly Executive 9(4), 2010, pp. 243-259.

[15] Dabbish, L., C. Stuart, J. Tsay, and J. Herbsleb, "Social coding in GitHub: transparency and collaboration in an open software repository", Proceedings of the ACM 2012 conference on computer supported cooperative work, ACM (2012), $1277-1286$.

[16] Degryse, C., "Shaping the World of Work in the Digital Economy”, SSRN Electronic Journal, 2017.

[17] Devaraj, S., and R. Kohli, "Performance impacts of information technology: Is actual usage the missing link?", Management science 49(3), 2003, pp. 273-289.

[18] Dong, J.Q., and W. Wu, "Business value of social media technologies: Evidence from online user innovation communities", The Journal of Strategic Information Systems 24(2), 2015, pp. 113-127. 
[19] Drnevich, P.L., and D.C. Croson, "Information Technology and Business-Level Strategy: Toward an Integrated Theoretical Perspective.”, Mis Quarterly 37(2), 2013, pp. 483509.

[20] Dumas, M., M.L. Rosa, J. Mendling, and H. Reijers, Fundamentals of Business Process Management 2nd Edition, Springer-Verlag, Berlin Heidelberg, 2018.

[21] Eisenmann, T.R., "Managing Proprietary and Shared Platforms.”, California Management Review 50(4), 2008, pp. $31-53$

[22] Erol, S., M. Granitzer, S. Happ, et al., "Combining BPM and social software: contradiction or chance?", Journal of Software Maintenance and Evolution: Research and Practice 22(6-7), 2010, pp. 449-476.

[23] Felsenstein, L., Community Memory: The First PublicAccess Social Media System, The MIT Press, 2016.

[24] Fisher, T., "ROI in social media: A look at the arguments", Journal of Database Marketing \& Customer Strategy Management 16(3), 2009, pp. 189-195.

[25] Garton, L., C. Haythornthwaite, and B. Wellman, "Studying online social networks", Journal of computer-mediated communication 3(1), 1997, pp. JCMC313.

[26] Granovetter, M., "The Strength of Weak Ties", The American Journal of Sociology 78(6), 1973, pp. 1360-1380.

[27] Hasan, H., and C.C. Pfaff, "The Wiki: an environment to revolutionise employees' interaction with corporate knowledge", Proceedings of the 20th CHISIG, ACM (2006), 377-380.

[28] Hevner, A., S. March, J. Park, and S. Ram, "Design Science in Information Systems Research", Management Information Systems Quarterly 28(1), 2008, pp. 75-105.

[29] Hitt, L., and E. Brynjolfsson, "Productivity, Business Profitability, and Consumer Surplus: Three Different Measures of Information Technology Value", Management Information Systems Quarterly 20(2), 1996, pp. 121-142.

[30] Howe, J., Crowdsourcing, Three Rivers Press, 2008

[31] Ifinedo, P., "Interactions between organizational size, culture, and structure and some IT factors in the context of ERP success assessment: an exploratory investigation", Journal of Computer Information Systems 47(4), 2007, pp. 28-44.

[32] Kane, G.C., "Enterprise social media: Current capabilities and future possibilities.", MIS Quarterly Executive 14(1), 2015.
[33] Kaplan, A.M., and M. Haenlein, "Users of the world, unite! The challenges and opportunities of Social Media", Business horizons 53(1), 2010, pp. 59-68.

[34] Kaplan, R.S., and D.P. Norton, The balanced scorecard: translating strategy into action, Harvard Business Press, 1996.

[35] Kates, A., and J.R. Galbraith, Designing your organization: Using the STAR model to solve 5 critical design challenges, John Wiley \& Sons, 2010.

[36] Kearns, G.S., and R. Sabherwal, "Antecedents and consequences of information systems planning integration", IEEE Transactions on Engineering Management 54(4), 2007, pp. 628-643.

[37] Kim, G., B. Shin, K.K. Kim, and H.G. Lee, "IT capabilities, process-oriented dynamic capabilities, and firm financial performance", Journal of the Association for Information Systems 12(7), 2011, pp. 487.

[38] Kirchner, K., and L. Razmerita, "Managing the Digital Knowledge Work with the Social Media Business Value Compass", Proceedings of the 52nd Hawaii International Conference on System Sciences, Hawaii International Conference on System Sciences (HICSS) (2019), 6438-6447.

[39] Kohli, R., and V. Grover, "Business value of IT: An essay on expanding research directions to keep up with the times", Journal of the association for information systems 9(1), 2008, pp. 1.

[40] Kwai Fun, I.P., "Weblogging: A study of social computing and its impact on organizations", Decision Support Systems 45(2), 2008, pp. 242-250.

[41] Li, C., A. Webber, and J. Cifuentes, "Making the business case for enterprise social networks", Altimeter report (February 2012), 2012.

[42] Littler, C.R., "Understanding taylorism", British Journal of Sociology, 1978, pp. 185-202.

[43] Majchrzak, A., S. Faraj, G.C. Kane, and B. Azad, "The contradictory influence of social media affordances on online communal knowledge sharing", Journal of Computer-Mediated Communication 19(1), 2013, pp. 38-55.

[44] Marjanovic, O., and M. Rothenhoefer, "Improving Knowledge-Intensive Business Processes Through Social Media”, AMCIS 2014 Proceedings, AISNET (2014).

[45] Melville, N., K. Kraemer, and V. Gurbaxani, "Information technology and organizational performance: An integrative model of IT business value", MIS quarterly 28(2), 2004, pp. 283-322. 
[46] Muller, M.J., J. Freyne, C. Dugan, D.R. Millen, and J. Thom-Santelli, "Return On Contribution (ROC): A metric for enterprise social software", In ECSCW 2009. Springer, 2009, 143-150.

[47] Okoli, C., and K. Schabram, "A guide to conducting a systematic literature review of information systems research", 2010.

[48] O'Reilly, T., What is Web 2.0 ?, O'Reilly, Newton, Massachusetts, 2009.

[49] Parker, G., M.W. van Alstyne, and S.P. Choudary, Platform Revolution: How Networked Markets are Transforming the Economy--and How to Make Them Work for You, Norton \& Company, New York, 2016.

[50] Pearson, N.M., "Fayolism as the Necessary Complement of Taylorism", American Political Science Review 39(1), 1945, pp. 68-80.

[51] Razmerita, L., K. Kirchner, and T. Nabeth, "Social Media in Organizations: Leveraging Personal and Collective Knowledge Processes", Journal of Organizational Computing and Electronic Commerce 24(1), 2014, pp. 74-93.

[52] Razmerita, L., K. Kirchner, and P. Nielsen, "What factors influence knowledge sharing in organizations? A social dilemma perspective of social media communication", Journal of Knowledge Management 20(6), 2016, pp. 1225-1246.

[53] Razmerita, L., K. Kirchner, and F. Sudzina, "Personal knowledge management: The role of Web 2.0 tools for managing knowledge at individual and organisational levels", Online information review 33(6), 2009, pp. 1021-1039.

[54] Recker, J., Scientific research in information systems: a beginner's guide, Springer Berlin Heidelberg, Berlin, Heidelberg, 2013.

[55] Richter, A., J. Heidemann, M. Klier, and S. Behrendt, "Success Measurement of Enterprise Social Networks.", Wirtschaftsinformatik 20, 2013, pp. 1-15.

[56] Scheer, A.W., ARIS-business process modeling, Springer Verlag, 2000.

[57] Schildmann, M., D. Bendig, and M. Brettel, "Creating Business Value Through Social Media: An Investigation of the Dynamic Relationship Between Social Media, Brand Equity and Firm Risk", pp. 13.

[58] Schlagwein, D., D. Schoder, and K. Fischbach, "Social Information Systems: Review, Framework, and Research Agenda", ICIS 2011 Proceedings, 2011.

[59] Schmidt, R., R. Alt, and S. Nurcan, "Social Information Systems", Proceedings of the 52nd Hawaii International Conference on System Sciences, (2019), 2642-2646.
[60] Schmidt, R., and S. Nurcan, "BPM and Social Software", Business Process Management Workshops, Springer Berlin Heidelberg (2009), 649-658.

[61] Schryen, G., "Revisiting IS business value research: what we already know, what we still need to know, and how we can get there", European Journal of Information Systems 22(2), 2013, pp. 139-169.

[62] Shirky, C., Cognitive surplus: Creativity and generosity in a connected age, Penguin UK, 2010.

[63] Surowiecki, J., The Wisdom of Crowds: : Why the Many Are Smarter Than the Few and How Collective Wisdom Shapes Business, Economies, Societies and Nations, Anchor, 2005.

[64] Tajvidi, R., and A. Karami, "The effect of social media on firm performance", Computers in Human Behavior, 2017, pp. S0747563217305514.

[65] Taylor, F.W., "The principles of scientific management", New York 202, 1911.

[66] Tsao, W.-C., M.-T. Hsieh, L.-W. Shih, and T.M. Lin, "Compliance with eWOM: The influence of hotel reviews on booking intention from the perspective of consumer conformity", International Journal of Hospitality Management 46, 2015, pp. 99-111.

[67] Vargo, S.L., and R.F. Lusch, "Evolving to a new dominant logic for marketing", Journal of Marketing 68(1), 2004, pp. 1-17.

[68] Wagner, H.-T., D. Beimborn, and T. Weitzel, "How social capital among information technology and business units drives operational alignment and IT business value", Journal of Management Information Systems 31(1), 2014, pp. 241272.

[69] Webster, J., and R.T. Watson, "Analyzing the Past to Prepare for the Future: Writing A", MIS Quarterly 26(2), 2002, pp. 494-508.

[70] Willcocks, L., E.A. Whitley, and C. Avgerou, "The ranking of top IS journals: a perspective from the London School of Economics", European Journal of Information Systems 17(2), 2008, pp. 163-168.

[71] Animation of Stakeholders: Understanding the Benefits of Social Networks to Organisations, European Commission, Brussels, 2012.

[72] "Digital Taylorism - Schumpeter", Economist, 2015. https://www.economist.com/business/2015/09/10/digital-taylorism 